\title{
A Few Integrable Couplings of Some Integrable Systems and $(2+1)$-Dimensional Integrable Hierarchies
}

\author{
Binlu Feng, ${ }^{1}$ Yufeng Zhang, ${ }^{2}$ and Huanhe Dong ${ }^{3}$ \\ ${ }^{1}$ School of Mathematics and Information Sciences, Weifang University, Weifang 261061, China \\ ${ }^{2}$ College of Sciences, China University of Mining and Technology, Xuzhou 221116, China \\ ${ }^{3}$ School of Mathematics, Shandong University of Science and Technology, Qingdao 266590, China \\ Correspondence should be addressed to Binlu Feng; fbl_1963@126.com
}

Received 25 April 2014; Accepted 20 July 2014; Published 14 August 2014

Academic Editor: Wen-Xiu Ma

Copyright (C) 2014 Binlu Feng et al. This is an open access article distributed under the Creative Commons Attribution License, which permits unrestricted use, distribution, and reproduction in any medium, provided the original work is properly cited.

Two high-dimensional Lie algebras are presented for which four $(1+1)$-dimensional expanding integrable couplings of the DAKNS hierarchy are obtained by using the Tu scheme; one of them is a united integrable coupling model of the D-AKNS hierarchy and the AKNS hierarchy. Then $(2+1)$-dimensional DS hierarchy is derived by using the TAH scheme; in particular, the integrable couplings of the DS hierarchy are obtained.

\section{Introduction}

Seeking new integrable systems has been an important aspect of soliton theory, and the methods for this are also versatile. For example, Magri [1] once proposed the Lax-pair method for generating $(1+1)$-dimensional integrable Hamiltonian equations. Tu [2] took use of some subalgebras of the Lie algebra $A_{1}$ to draw a beautiful picture for generating integrable Hamiltonian equation hierarchies with $1+1$ dimensions, and in particular, the AKNS hierarchy, the KN hierarchy, the WKI hierarchy, and others were obtained under the frame of zero curvature equations. Ma [3] called the method the Tu scheme, and $\mathrm{Ma}$ also obtained a series of interesting results in $[4-6] . \mathrm{Hu}[7,8]$ further generalized the trace identity of Tu scheme to get the supertrace identity for generating superintegrable Hamiltonian systems which has extended the applications of the Tu scheme. Fan $[9,10]$ applied the Tu scheme not only to have obtained some new integrable hierarchies of equations, but also to have obtained their some geometric properties. Various Lax pairs can be introduced by some reductions of the self-dual Yang-Mills equations. For example, Chakravarty et al. [11] introduced symmetries and gauge transformations as well as isospectral problems by reducing the self-dual Yang-Mills equations to have obtained $(1+1)$ and $(2+1)$-dimensional integrable systems. Ablowitz et al.
[12] introduced some different Lax pairs by reductions of the self-dual Yang-Mills equations and further obtained some celebrated $(1+1)$ - and $(2+1)$-dimensional integrable equations, including the $\mathrm{KdV}$ equation and the $\mathrm{KP}$ equation. It is remarkable that generating $(2+1)$-dimensional integrable hierarchies is more difficult than $(1+1)$-dimensional ones. The papers $[11,12]$ were proceeded by introducing a Lie algebra $g=\left\{a_{0}+a_{1}(\partial / \partial y)+a_{2}\left(\partial^{2} / \partial y^{2}\right)\right\}$, where $a_{i}$ belong to a ring of $n \times n$ complex matrix functions of $y, x$, and $t$, while Tu et al. [13] proposed an efficient and direct approach for generating $(2+1)$-dimensional equation hierarchies by introducing a residue operator, which was called by us the TAH scheme. Because few people further discussed the scheme and investigated other new $(2+1)$-dimensional integrable hierarchies, we would like to recall the scheme and apply it to produce new $(2+1)$-dimensional hierarchies of equations along with multipotential functions. First of all, we recall some related notations.

Let $\mathscr{A}$ be an associative algebra over the field $\mathscr{R}$. An operator $\partial: \mathscr{A} \rightarrow \mathscr{A}$ satisfies that

$$
\partial(\alpha f+\beta g)=\alpha \partial f+\beta \partial g, \quad \partial(f g)=(\partial f) g+f(\partial g),
$$

where $\alpha, \beta \in \mathscr{R} ; f, g \in \mathscr{A}$. 
Introduce an associative algebra $\mathscr{A}[\xi]$ consisting of the pseudodifferential operator $\sum_{-\infty}^{N} a_{i} \xi^{i}$, where the coefficients $a_{i} \in \mathscr{A}$, and $\xi$ is an operator given by

$$
\xi f=f \xi+\left(\partial_{y} f\right), \quad f \in \mathscr{A} .
$$

It can be verified that [13]

$$
\begin{gathered}
\xi^{n} f=\sum_{i \geq 0}\left(\begin{array}{c}
n \\
i
\end{array}\right)\left(\partial^{i} f\right) \xi^{n-i}, \quad n \in \mathbf{Z}, \\
f^{n}=\sum_{i \geq 0}(-1)^{i}\left(\begin{array}{c}
n \\
i
\end{array}\right) \xi^{n-i}\left(\partial^{i} f\right), \quad n \in \mathbf{Z} .
\end{gathered}
$$

Besides, a residue operator is given by

$$
R: \mathscr{A}[\xi] \longrightarrow \mathscr{A}, \quad R\left(\sum a_{i} \xi^{i}\right)=a_{-1} .
$$

Based on the above, we recall the TAH scheme as follows.

(1) Fix a matrix operator $U=U(\lambda+\xi, u) \in \mathscr{A}[\xi]$, where $u=\left(u_{1}, \ldots, u_{p}\right)^{T}$.

(2) Solving the matrix-operator equation

$$
V_{x}=[U, V]
$$

leads to a recursion relation among $V_{n}$, where $V=$ $\sum V_{n} \lambda^{-n}$, from which we will obtain a recursion relation among $g^{(n)} \equiv\left(g_{1}^{(n)}, \ldots, g_{p}^{(n)}\right)^{T}$, where $g_{i}^{(n)}$ comes from the expansion

$$
\left\langle V, \frac{\partial U}{\partial u_{i}}\right\rangle=\sum_{n} g_{i}^{(n)} \lambda^{-n}
$$

where $\langle a, b\rangle=\operatorname{tr} R(a b)$.

(3) Try to find an operator $J$ and form the hierarchy

$$
u_{t_{n}}=J g^{(n)}
$$

(4) Employing the trace identity [13]

$$
\frac{\delta}{\delta u_{i}}\left\langle V, \frac{\partial U}{\partial \lambda}\right\rangle=\left(\lambda^{-\gamma} \frac{\partial}{\partial \lambda} \lambda^{\gamma}\right)\left\langle V, \frac{\partial U}{\partial u_{i}}\right\rangle
$$

could obtain the Hamiltonian structure of the $(2+1)$ dimensional hierarchy (8).

In the paper, we want to employ the TAH scheme to generalize $2 \times 2$ matrix operators which are presented in $[13,14]$ to $4 \times 4$ cases by using the constructed higher-dimensional Lie algebras so that $(2+1)$-dimensional equation hierarchies could be generated, whose Hamiltonian structure is also obtained by the trace identity (9). For that sake, we first consider the D-AKNS hierarchy which is derived by our Lie algebra, and then we expand the D-AKNS hierarchy to obtain four different integrable models with 4,5 , and 6 potential functions, respectively. In particular, one of them can reduce to the well-known AKNS hierarchy.

\section{The D-AKNS Hierarchy and Its Expanding Integrable Models}

A Lie subalgebra of the Lie algebra $A_{1}$ presents that [14]

$$
L=\operatorname{span}\left\{h_{1}, h_{2}, h_{3}, h_{4}\right\} \text {, }
$$

where

$$
\begin{array}{cc}
h_{1}=\left(\begin{array}{ll}
1 & 0 \\
0 & 0
\end{array}\right), & h_{2}=\left(\begin{array}{ll}
0 & 1 \\
0 & 0
\end{array}\right), \quad h_{3}=\left(\begin{array}{ll}
0 & 0 \\
1 & 0
\end{array}\right), \\
h_{4}=\left(\begin{array}{ll}
0 & 0 \\
0 & 1
\end{array}\right) . &
\end{array}
$$

A loop algebra is defined by

$$
\widetilde{L}=\operatorname{span}\left\{h_{1}(n), h_{2}(n), h_{3}(n), h_{4}(n)\right\},
$$

where $h_{i}(n)=h_{i} \lambda^{n}, n \in \mathbf{Z}$, which have the commutative relations

$$
\begin{aligned}
& {\left[h_{1}(m), h_{2}(n)\right]=h_{2}(m+n),} \\
& {\left[h_{1}(m), h_{3}(n)\right]=-h_{3}(m+n), \quad\left[h_{1}(m), h_{4}(n)\right]=0,} \\
& {\left[h_{2}(m), h_{3}(n)\right]=h_{1}(m+n)-h_{4}(m+n),} \\
& {\left[h_{2}(m), h_{4}(n)\right]=h_{2}(m+n),} \\
& {\left[h_{3}(m), h_{4}(n)\right]=-h_{3}(m+n),} \\
& \qquad m, n \in \mathbf{Z} .
\end{aligned}
$$

Paper [14] had derived the D-AKNS hierarchy. Now we employ the loop algebra $\widetilde{L}$ to rederive it again by the Tu scheme to make the paper self-contained by following $\mathrm{Tu}$ idea, and then two exlargening Lie algebras of the Lie algebra $L$ can be used to generate the expanding integrable models, specially, containing the $(2+1)$-dimensional expanding equation hierarchies.

Set

$$
\begin{aligned}
& U=h_{4}(1)+s h_{4}(0)+q h_{2}(0)+r h_{3}(0), \\
& V=\sum_{m \geq 0}\left(\sum_{i=1}^{4} V_{i m} h_{i}(-m)\right) .
\end{aligned}
$$

A solution to (14) for $V$ is given by

$$
\begin{aligned}
& V_{1 m, x}=q V_{3 m}-r V_{2 m}=-V_{4 m, x}, \\
& V_{2, m+1}=-V_{2 m, x}-s V_{2 m}-q V_{1 m}+q V_{4 m}, \\
& V_{3, m+1}=V_{3 m, x}-s V_{3 m}-r V_{1 m}+r V_{4 m} .
\end{aligned}
$$

Letting

$$
V^{(n)}=\sum_{m=0}^{n}\left(\sum_{i=1}^{4} V_{i m} h_{i}(n-m)\right)+\left(V_{1 n}-V_{4 n}\right) h_{4}(0),
$$


a direct calculation yields that

$$
\begin{aligned}
-V_{x}^{(n)}+\left[U, V^{(n)}\right]= & \left(V_{4 n, x}-V_{1 n, x}\right) h_{4}(1)+\left(-V_{2 n, x}-s V_{2 n}\right) \\
& \times h_{2}(0)-\left(V_{3 n, x}-s V_{3 n}\right) h_{3}(0)
\end{aligned}
$$

According to the Tu scheme, the zero curvature equation

$$
U_{t_{n}}-V_{x}^{(n)}+\left[U, V^{(n)}\right]=0
$$

admits that

$$
\begin{aligned}
& q_{t_{n}}=V_{2 n, x}+s V_{2 n}, \\
& r_{t_{n}}=V_{3 n, x}-s V_{3 n}, \\
& s_{t_{n}}=-2 V_{4 n, x} .
\end{aligned}
$$

Denoting $u=(q, r, s)^{T}$, then we have

$$
u_{t_{n}}=\left(\begin{array}{ccc}
0 & \partial+s & 0 \\
\partial-s & 0 & 0 \\
0 & 0 & -2 \partial
\end{array}\right)\left(\begin{array}{c}
V_{3 n} \\
V_{2 n} \\
V_{4 n}
\end{array}\right)=J_{1}\left(\begin{array}{c}
V_{3 n} \\
V_{2 n} \\
V_{4 n}
\end{array}\right),
$$

which is just right the D-AKNS hierarchy. Obviously, $J_{1}$ is a Hamiltonian operator. The Hamiltonian structure of (20) can be obtained by the trace identity in [2]:

$$
\frac{\delta}{\delta u}\left(4 V_{4}\right)=\left(\lambda^{-\gamma} \frac{\partial}{\partial \lambda} \lambda^{\gamma}\right)\left(\begin{array}{c}
V_{3} \\
V_{2} \\
V_{4}
\end{array}\right)
$$

Comparing the coefficients of $\lambda^{-n-1}$ gives

$$
\frac{\delta}{\delta u}\left(4 V_{4, n+1}\right)=(-n+\gamma)\left(\begin{array}{c}
V_{3 n} \\
V_{2 n} \\
V_{4 n}
\end{array}\right) .
$$

If set $V_{2,0}=V_{3,0}=V_{4,0}=0, V_{1,0}=$ constant, we can get some explicit values from (15) $V_{2,1}=-\alpha q, V_{3,1}=-\alpha r$, and $V_{1,1}=$ $0, \ldots$.

In terms of the initial values, we can get $\gamma=0$. Thus, we obtain the Hamiltonian structure of (20)

$$
u_{t_{n}}=J_{1} \frac{\delta H_{n}}{\delta u}
$$

where $H_{n}=-4 V_{4, n+1} / n$.

In what follows, we discuss four different expanding integrable models of the D-AKNS hierarchy. With the help of the Lie algebra $L$, we introduce an 8-dimensional Lie algebra

$$
G_{1}=\operatorname{span}\left\{f_{1}, \ldots, f_{8}\right\},
$$

where

$$
\begin{gathered}
f_{1}=\left(\begin{array}{cc}
h_{1} & 0 \\
0 & h_{1}
\end{array}\right), \quad f_{2}=\left(\begin{array}{cc}
h_{4} & 0 \\
0 & h_{4}
\end{array}\right), \quad f_{3}=\left(\begin{array}{cc}
h_{2} & 0 \\
0 & h_{2}
\end{array}\right), \\
f_{4}=\left(\begin{array}{cc}
h_{3} & 0 \\
0 & h_{3}
\end{array}\right), \quad f_{5}=\left(\begin{array}{ll}
0 & h_{1} \\
0 & h_{1}
\end{array}\right), \quad f_{6}=\left(\begin{array}{ll}
0 & h_{4} \\
0 & h_{4}
\end{array}\right), \\
f_{7}=\left(\begin{array}{ll}
0 & h_{2} \\
0 & h_{2}
\end{array}\right), \quad f_{8}=\left(\begin{array}{ll}
0 & h_{3} \\
0 & h_{3}
\end{array}\right),
\end{gathered}
$$

which possesses the commutative relations

$$
\begin{aligned}
& {\left[f_{1}, f_{2}\right]=0, \quad\left[f_{1}, f_{3}\right]=f_{3}, \quad\left[f_{1}, f_{4}\right]=-f_{4},} \\
& {\left[f_{1}, f_{5}\right]=\left[f_{1}, f_{6}\right]=0, \quad\left[f_{1}, f_{7}\right]=f_{7},} \\
& {\left[f_{1}, f_{8}\right]=-f_{8}, \quad\left[f_{2}, f_{3}\right]=-f_{3}, \quad\left[f_{2}, f_{4}\right]=f_{4},} \\
& {\left[f_{2}, f_{5}\right]=\left[f_{2}, f_{6}\right]=0, \quad\left[f_{2}, f_{7}\right]=-f_{7},} \\
& {\left[f_{2}, f_{8}\right]=f_{8}, \quad\left[f_{3}, f_{4}\right]=f_{1}-f_{2}, \quad\left[f_{3}, f_{5}\right]=-f_{7},} \\
& {\left[f_{3}, f_{6}\right]=f_{7}, \quad\left[f_{3}, f_{7}\right]=0,} \\
& {\left[f_{3}, f_{8}\right]=f_{5}-f_{6}, \quad\left[f_{4}, f_{5}\right]=f_{8}, \quad\left[f_{4}, f_{6}\right]=-f_{8},} \\
& {\left[f_{4}, f_{7}\right]=f_{6}-f_{5}, \quad\left[f_{4}, f_{8}\right]=\left[f_{5}, f_{6}\right]=0,} \\
& {\left[f_{5}, f_{7}\right]=f_{7}, \quad\left[f_{5}, f_{8}\right]=-f_{8}, \quad\left[f_{6}, f_{7}\right]=-f_{7},} \\
& {\left[f_{6}, f_{8}\right]=f_{8}, \quad\left[f_{7}, f_{8}\right]=f_{5}-f_{6} .}
\end{aligned}
$$

A loop algebra can be defined as

$$
\begin{gathered}
f_{i}(n)=f_{i} \lambda^{n}, \quad i=1,2, \ldots, 8 \\
{\left[f_{i}(m), f_{j}(n)\right]=\left[f_{i}, f_{j}\right] \lambda^{m+n}, \quad 1 \leq i, \quad j \leq 8 ; m, n \in \mathbf{Z} .}
\end{gathered}
$$

Now we utilize the loop algebra to introduce the following Lax pair:

$$
\begin{gathered}
U=f_{2}(1)+s f_{2}(0)+q f_{3}(0)+r f_{4}(0)+f_{6}(1) \\
+u_{1} f_{7}(0)+u_{2} f_{8}(0)+u_{3} f_{5}(0), \\
V=\sum_{m \geq 0}\left(V_{1 m} f_{1}(-m)+V_{2 m} f_{3}(-m)+V_{3 m} f_{4}(-m)\right. \\
\left.+V_{4 m} f_{2}(-m)+\sum_{i=5}^{8} V_{i m} f_{i}(-m)\right) .
\end{gathered}
$$

According to the Tu scheme, we first need to solve the matrix equation for $V$ :

$$
V_{x}=[U, V]
$$

which admits the first part equations (15) and the second part ones as follows:

$$
\begin{aligned}
\left(V_{5 m}\right)_{x}= & u_{1} V_{3 m}-u_{2} V_{2 m}-\left(r+u_{2}\right) V_{7 m}+\left(q+u_{1}\right) V_{8 m} \\
= & -\left(V_{6 m}\right)_{x} \\
\left(V_{7 m}\right)_{x}= & -2 V_{7, m+1}+\left(-s+u_{3}\right) V_{7 m}+\left(q+u_{1}\right) V_{6 m} \\
& -\left(q+u_{1}\right) V_{5 m}-u_{1} V_{1 m}+u_{1} V_{4 m}+u_{3} V_{2 m}-V_{2, m+1}, \\
\left(V_{8 m}\right)_{x}= & 2 V_{8, m+1}+\left(s-u_{3}\right) V_{8 m}-\left(r+u_{2}\right) V_{6 m} \\
& +\left(r+u_{2}\right) V_{5 m}+u_{2} V_{1 m}-u_{2} V_{4 m}-u_{3} V_{3 m}+V_{3, m+1} .
\end{aligned}
$$


Taking

$$
\begin{gathered}
V^{(n)}=\sum_{m=0}^{n}\left(V_{1 m} f_{1}(n-m)+V_{2 m} f_{3}(n-m)+V_{3 m} f_{4}(n-m)\right. \\
\left.+V_{4 m} f_{2}(n-m)+\sum_{i=5}^{8} V_{i m} f_{i}(-m) \lambda^{n}\right) \\
+\left(V_{1 n}-V_{4 n}\right) f_{2}(0)+\left(V_{6 n}-V_{5 n}\right) f_{5}(0),
\end{gathered}
$$

one infers that

$$
\begin{aligned}
& -V_{x}^{(n)}+\left[U, V^{(n)}\right] \\
& =-\left(V_{1 n}-V_{4 n}\right)_{x} f_{2}(0)+\left[V_{2, n+1}+q\left(V_{1 n}-V_{4 n}\right)\right] f_{3}(0) \\
& -\left[V_{3, n+1}-r\left(V_{6 n}-V_{5 n}\right)\right] f_{4}(0)-\left(V_{6 n}-V_{5 n}\right)_{x} f_{5}(0) \\
& +\left[V_{2, n+1}+2 V_{7, n+1}+u_{1}\left(V_{1 n}-V_{4 n}\right)\right. \\
& \left.\quad-\left(q+u_{1}\right)\left(V_{6 n}-V_{5 n}\right)\right] f_{7}(0) \\
& +\left[-2 V_{8, n+1}-V_{3, n+1}-u_{2}\left(V_{1 n}-V_{4 n}\right)\right. \\
& \left.\quad+\left(r+u_{2}\right)\left(V_{6 n}-V_{5 n}\right)\right] f_{8}(0) .
\end{aligned}
$$

Thus, the zero curvature equation

$$
U_{t_{n}}-V_{x}^{(n)}+\left[U, V^{(n)}\right]=0
$$

gives rise to

$$
u_{t_{n}}=\left(\begin{array}{c}
q \\
r \\
s \\
u_{1} \\
u_{2} \\
u_{3}
\end{array}\right)_{t_{n}}=\left(\begin{array}{c}
\left(V_{2 n}\right)_{x}+s V_{2 n} \\
\left(V_{3 n}\right)_{x}-s V_{3 n} \\
-2\left(V_{4 n}\right)_{x} \\
\left(V_{7 n}\right)_{x}+s V_{7 n}-u_{3}\left(V_{2 n}+V_{7 n}\right) \\
\left(V_{8 n}\right)_{x}-s V_{8 n}+u_{3}\left(V_{3 n}+V_{8 n}\right) \\
-2\left(V_{5 n}\right)_{x}
\end{array}\right) .
$$

When $u_{1}=u_{2}=u_{3}=0$, the hierarchy (34) reduces to the D-AKNS hierarchy.

When $s=u_{3}=0$, (34) reduces to the following expanding integrable system of the AKNS hierarchy:

$$
\bar{u}_{t_{n}}=\left(\begin{array}{c}
q \\
r \\
u_{1} \\
u_{2}
\end{array}\right)_{t_{n}}=\left(\begin{array}{cccc}
0 & \partial_{x} & 0 & 0 \\
\partial_{x} & 0 & 0 & 0 \\
0 & 0 & 0 & \partial_{x} \\
0 & 0 & \partial_{x} & 0
\end{array}\right)\left(\begin{array}{l}
V_{3 n} \\
V_{2 n} \\
V_{8 n} \\
V_{7 n}
\end{array}\right)=J\left(\begin{array}{l}
V_{3 n} \\
V_{2 n} \\
V_{8 n} \\
V_{7 n}
\end{array}\right) .
$$

In particular, setting $u_{1}=u_{2}=0$, (35) again reduces to the known AKNS hierarchy

$$
\tilde{u}_{t_{n}}=\left(\begin{array}{l}
q \\
r
\end{array}\right)_{t_{n}}=\left(\begin{array}{cc}
0 & \partial_{x} \\
\partial_{x} & 0
\end{array}\right)\left(\begin{array}{l}
V_{3 n} \\
V_{2 n}
\end{array}\right) .
$$

Therefore, the hierarchy (34) is a united expanding integrable model of the D-AKNS hierarchy, the AKNS hierarchy, and the hierarchy (36). It is easy to see that (34) is different from that presented in [14], which indicates one of the merits for seeking multipotential expanding integrable hierarchies. However, the Hamiltonian structure of (34) cannot be obtained by the variational identity [15], which makes us feel confusions.

In order to get the second expanding integrable model of the D-AKNS hierarchy, we first introduce the following Lie algebra:

$$
G_{2}=\operatorname{span}\left\{g_{1}, \ldots, g_{8}\right\}
$$

where

$$
\begin{array}{cc}
g_{1}=f_{1}, & g_{2}=f_{2}, \quad g_{3}=f_{3}, \quad g_{4}=f_{4}, \\
g_{5}=\left(\begin{array}{cc}
0 & h_{1} \\
h_{1} & 0
\end{array}\right), & g_{6}=\left(\begin{array}{cc}
0 & h_{4} \\
h_{4} & 0
\end{array}\right), \quad g_{7}=\left(\begin{array}{cc}
0 & h_{2} \\
h_{2} & 0
\end{array}\right), \\
g_{8}=\left(\begin{array}{cc}
0 & h_{3} \\
h_{3} & 0
\end{array}\right), &
\end{array}
$$

which has the following commutative relations:

$$
\begin{aligned}
& {\left[g_{1}, g_{2}\right]=0, \quad\left[g_{1}, g_{3}\right]=g_{3}, \quad\left[g_{1}, g_{4}\right]=-g_{4},} \\
& {\left[g_{2}, g_{3}\right]=-g_{3}, \quad\left[g_{2}, g_{4}\right]=g_{4}, \quad\left[g_{3}, g_{4}\right]=g_{1}-g_{2},} \\
& {\left[g_{1}, g_{5}\right]=\left[g_{1}, g_{6}\right]=0, \quad\left[g_{1}, g_{7}\right]=g_{7},} \\
& {\left[g_{1}, g_{8}\right]=-g_{8}, \quad\left[g_{2}, g_{5}\right]=\left[g_{2}, g_{6}\right]=0,} \\
& {\left[g_{2}, g_{7}\right]=-g_{7}, \quad\left[g_{2}, g_{8}\right]=g_{8}, \quad\left[g_{3}, g_{5}\right]=-g_{7},} \\
& {\left[g_{3}, g_{6}\right]=g_{7}, \quad\left[g_{3}, g_{7}\right]=0, \quad\left[g_{3}, g_{8}\right]=g_{5}-g_{6},} \\
& {\left[g_{4}, g_{5}\right]=g_{8}, \quad\left[g_{4}, g_{6}\right]=-g_{8}, \quad\left[g_{4}, g_{7}\right]=g_{6}-g_{5},} \\
& {\left[g_{4}, g_{8}\right]=\left[g_{5}, g_{6}\right]=0, \quad\left[g_{7}\right]=g_{3},} \\
& {\left[g_{5}, g_{8}\right]=-g_{4}, \quad\left[g_{6}, g_{7}\right]=-g_{3}, \quad\left[g_{6}, g_{8}\right]=g_{4},} \\
& {\left[g_{7}, g_{8}\right]=g_{1}-g_{2} .}
\end{aligned}
$$

Now we let us compare the Lie algebras $G_{1}$ and $G_{2}$. Setting

$$
\begin{aligned}
& G_{11}=\operatorname{span}\left\{f_{1}, f_{2}, f_{3}, f_{4}\right\}, \\
& G_{12}=\operatorname{span}\left\{f_{5}, f_{6}, f_{7}, f_{8}\right\},
\end{aligned}
$$

we find that

$$
G_{1}=G_{11} \oplus G_{12}, \quad\left[G_{11}, G_{12}\right] \subset G_{12} .
$$

Condition (41) is a sufficient condition for generating integrable couplings.

If we set

$$
\begin{aligned}
& G_{21}=\operatorname{span}\left\{g_{1}, g_{2}, g_{3}, g_{4}\right\}, \\
& G_{22}=\operatorname{span}\left\{g_{5}, g_{6}, g_{7}, g_{8}\right\},
\end{aligned}
$$


it is easy to see that

$$
G_{2}=G_{21}+G_{22}, \quad\left[G_{21}, G_{22}\right] \text { not in } G_{22} .
$$

Equation (43) is different from (41) because the subalgebra $G_{22}$ is a semisimple Lie algebra, while $G_{12}$ is not. Therefore, if we start the Lie algebra $G_{2}$ to introduce Lax pairs, some new integrable hierarchies could be generated by employing the Tu scheme, which were not integrable couplings of some known integrable systems. In what follows, we want to make use of a loop algebra of the Lie algebra $G_{2}$ to introduce a Lax pair so that the second expanding hierarchy of the D-AKNS hierarchy could be derived by the Tu scheme. A loop algebra of the Lie algebra $G_{2}$ is defined as

$$
\widetilde{G}_{2}=\operatorname{span}\left\{g_{1}(n), \ldots, g_{8}(n)\right\},
$$

where

$$
\begin{gathered}
g_{i}(n)=g_{i} \lambda^{n}, \quad i=1,2, \ldots, 8 ; \\
{\left[g_{i}(m), g_{j}(n)\right]=\left[g_{i}, g_{j}\right] \lambda^{m+n}, \quad 1 \leq i, \quad j \leq 8 ; m, n \in \mathbf{Z} .}
\end{gathered}
$$

\section{Consider a Lax pair}

$$
\begin{aligned}
& U= g_{2}(1)+s g_{2}(0)+q g_{3}(0)+r g_{4}(0)+v_{1} g_{7}(0) \\
&+v_{2} g_{8}(0)+v_{3} g_{5}(0), \\
& V=\sum_{m \geq 0}\left(V_{1 m} g_{1}(-m)+V_{2 m} g_{3}(-m)+V_{3 m} g_{4}(-m)\right. \\
&\left.+V_{4 m} g_{2}(-m)+\sum_{i=5}^{8} V_{i m} g_{i}(-m)\right) .
\end{aligned}
$$

Solving the stationary zero curvature equation

$$
V_{x}=[U, V]
$$

yields that

$$
\begin{aligned}
\left(V_{1 m}\right)_{x}= & q V_{3 m}-r V_{2 m}+v_{1} V_{8 m}-v_{2} V_{7 m}=-\left(V_{4 m}\right)_{x}, \\
V_{2, m+1}= & \left(V_{2 m}\right)_{x}-s V_{2 m}-q V_{1 m}+q V_{4 m}-v_{1} V_{5 m} \\
& +v_{1} V_{6 m}+v_{3} V_{7 m}, \\
\left(V_{3 m}\right)_{x}= & V_{3, m+1}+s V_{3 m}+r V_{1 m}-r V_{4 m}+v_{2} V_{5 m} \\
& -v_{2} V_{6 m}-v_{3} V_{8 m}, \\
\left(V_{5 m}\right)_{x}= & q V_{8 m}-r V_{7 m}+v_{1} V_{3 m}-v_{2} V_{2 m}=-\left(V_{6 m}\right)_{x}, \\
V_{7, m+1}= & \left(V_{7 m}\right)_{x}-q V_{5 m}+q V_{6 m}-v_{1} V_{1 m}+v_{1} V_{4 m} \\
& +v_{3} V_{2 m}-s V_{7 m}, \\
\left(V_{8 m}\right)_{x}= & V_{8, m+1}+s V_{8 m}+r V_{5 m}-r V_{6 m}+v_{2} V_{1 m} \\
& +v_{2} V_{1 m}-v_{2} V_{4 m}-v_{3} V_{3 m} .
\end{aligned}
$$

Taking

$$
\begin{gathered}
V^{(n)}=\sum_{m=0}^{n}\left(V_{1 m} g_{1}(n-m)+V_{2 m} g_{3}(n-m)+V_{3 m} g_{4}(n-m)\right. \\
\left.\quad+V_{4 m} g_{2}(n-m)+\sum_{i=5}^{8} V_{i m} g_{i}(n-m)\right) \\
+\left(V_{1 n}-V_{4 n}\right) f_{2}(0)+\left(V_{6 n}-V_{5 n}\right) f_{5}(0),
\end{gathered}
$$

similar to the above discussion, we obtain the integrable hierarchy

$$
u_{t_{n}}=\left(\begin{array}{c}
q \\
r \\
s \\
v_{1} \\
v_{2} \\
v_{3}
\end{array}\right)_{t_{n}}=\left(\begin{array}{c}
\left(V_{2 n}\right)_{x}+s V_{2 n}-v_{3} V_{7 n} \\
\left(V_{3 n}\right)_{x}-s V_{3 n}+v_{3} V_{8 n} \\
-2\left(V_{4 n}\right)_{x} \\
\left(V_{7 n}\right)_{x}+s V_{7 n}-v_{3} V_{2 n} \\
\left(V_{8 n}\right)_{x}-s V_{8 n}+v_{3} V_{3 n} \\
-2\left(V_{5 n}\right)_{x}
\end{array}\right) .
$$

Obviously, (50) is different from (34). However, when $v_{3}=$ $u_{3}=0$ in (50) and (34), respectively, they all reduce to the same equation hierarchy (below we will see it). When $v_{1}=$ $v_{2}=v_{3}=0$, (50) reduces to the D-AKNS hierarchy. Therefore, (50) is the second expanding integrable model of the D-AKNS hierarchy.

In the following, we consider the Hamiltonian structure of (50). Through direct calculation, we get that

$$
\begin{array}{rlr}
\left\langle V, U_{q}\right\rangle=2 V_{3}, & \left\langle V, U_{r}\right\rangle=2 V_{2}, \quad\left\langle V, U_{s}\right\rangle=2 V_{4}, \\
\left\langle V, U_{v_{1}}\right\rangle=2 V_{8}, & \left\langle V, U_{v_{2}}\right\rangle=2 V_{7}, \quad\left\langle V, U_{v_{3}}\right\rangle=2 V_{5}, \\
\left\langle V, U_{\lambda}\right\rangle=2 V_{4}, &
\end{array}
$$

and here $\langle a, b\rangle=\operatorname{tr}(a b), V_{i}=\sum_{m \geq 0} V_{i m} \lambda^{-m}, i=1,2, \ldots, 8$.

Substituting the above results into the trace identity gives

$$
\frac{\delta}{\delta u}\left(2 V_{4}\right)=\lambda^{-\gamma} \frac{\partial}{\partial \lambda} \lambda^{\gamma}\left(\begin{array}{c}
2 V_{3} \\
2 V_{2} \\
2 V_{4} \\
2 V_{8} \\
2 V_{7} \\
2 V_{5}
\end{array}\right) .
$$

Comparing the coefficients of $\lambda^{-n-1}$ leads to

$$
\frac{\delta}{\delta u}\left(2 V_{4, n+1}\right)=(-n+\gamma)\left(\begin{array}{c}
2 V_{3 n} \\
2 V_{2 n} \\
2 V_{4 n} \\
2 V_{8 n} \\
2 V_{7 n} \\
2 V_{5 n}
\end{array}\right) .
$$


Similar to the above discussion, we get $\gamma=0$. Thus, one gets that

$$
\left(\begin{array}{l}
2 V_{3 n} \\
2 V_{2 n} \\
2 V_{4 n} \\
2 V_{8 n} \\
2 V_{7 n} \\
2 V_{5 n}
\end{array}\right)=\frac{\delta H_{n}}{\delta u}, \quad H_{n}=-\frac{2 V_{4, n+1}}{n}
$$

Equation (50) can be writtten as

$$
\begin{aligned}
u_{t_{n}}=\left(\begin{array}{c}
q \\
r \\
s \\
v_{1} \\
v_{2} \\
v_{3}
\end{array}\right) \\
=\left(\begin{array}{cccccc}
t_{n} & \frac{\partial+s}{2} & 0 & 0 & -\frac{v_{3}}{2} & 0 \\
\frac{\partial-s}{2} & 0 & 0 & \frac{v_{3}}{2} & 0 & 0 \\
0 & 0 & -\partial & 0 & 0 & 0 \\
0 & -\frac{v_{3}}{2} & 0 & 0 & \frac{\partial+s}{2} & 0 \\
\frac{v_{3}}{2} & 0 & 0 & \frac{\partial-s}{2} & 0 & 0 \\
0 & 0 & 0 & 0 & 0 & -\partial
\end{array}\right)\left(\begin{array}{l}
2 V_{3 n} \\
2 V_{2 n} \\
2 V_{4 n} \\
2 V_{8 n} \\
2 V_{7 n} \\
2 V_{5 n}
\end{array}\right) \\
=J_{2} \frac{\delta H_{n}}{\delta u} . \\
\end{aligned}
$$

When $v_{3}=0$, (55) reduces to

$$
\begin{aligned}
& \bar{u}_{t_{n}}=\left(\begin{array}{c}
q \\
r \\
s \\
v_{1} \\
v_{2}
\end{array}\right)_{t_{n}}=\left(\begin{array}{c}
\left(V_{2 n}\right)_{x}+s V_{2 n} \\
(V-3 n)_{x}-s V_{3 n} \\
-2\left(V_{4 n}\right)_{x} \\
\left(V_{7 n}\right)_{x}+s V_{7 n} \\
\left(V_{8 n}\right)_{x}-s V_{8 n}
\end{array}\right) \\
& =\left(\begin{array}{ccccc}
0 & \frac{\partial+s}{2} & 0 & 0 & 0 \\
\frac{\partial-s}{2} & 0 & 0 & 0 & 0 \\
0 & 0 & -\partial & 0 & 0 \\
0 & 0 & 0 & 0 & \frac{\partial+s}{2} \\
0 & 0 & 0 & \frac{\partial-s}{2} & 0
\end{array}\right)\left(\begin{array}{c}
2 V_{3 n} \\
2 V_{2 n} \\
2 V_{4 n} \\
2 V_{8 n} \\
2 V_{7 n}
\end{array}\right) \\
& =J_{3}\left(\begin{array}{l}
2 V_{3 n} \\
2 V_{2 n} \\
2 V_{4 n} \\
2 V_{8 n} \\
2 V_{7 n}
\end{array}\right)
\end{aligned}
$$

which is also an expanding integrable model of the D-AKNS hierarchy.

\section{The $(2+1)$-Dimensional D-AKNS Hierarchy and Its Expanding Models}

In the section, we will apply the TAH scheme to deduce the $(2+1)$-dimensional D-AKNS hierarchy and its various expanding models, from which we obtain the united $(2+1)$ dimensional model of the $(2+1)$-dimensional D-AKNS hierarchy and the Davey-Stewartson (DS) hierarchy. The key idea presents that $4 \times 4$ matrix operators are introduced by our Lie algebras which extend the $2 \times 2$ cases in [13]. The work is worth going on because there are few people applying the method to generate new $(2+1)$-dimensional integrable hierarchies. First of all, we deduce the $(2+1)$-dimensional D-AKNS hierarchy. Set

$$
U=\left(\begin{array}{cc}
0 & q \\
r & \lambda+\stackrel{\xi}{\xi}+s
\end{array}\right), \quad V=\left(\begin{array}{ll}
A & B \\
C & D
\end{array}\right),
$$

where

$$
\begin{aligned}
A=\sum_{m \geq 0} A_{m} \lambda^{-m}, \quad B & =\sum_{m \geq 0} B_{m} \lambda^{-m}, \quad C=\sum_{m \geq 0} C_{m} \lambda^{-m}, \\
D & =\sum_{m \geq 0} D_{m} \lambda^{-m} .
\end{aligned}
$$

According to the TAH scheme, we solve the equation of the matrix operators (6) which gives rise to

$$
\begin{gathered}
A_{x}=q C-B r, \\
B_{x}=q D-A q-\lambda B-B \xi-B s, \\
C_{x}=r A-D r+\lambda C+\xi C+s C, \\
D_{x}=r B-C q+\xi D+s D-D \xi-D s .
\end{gathered}
$$

Inserting (58) into (59) reads

$$
\begin{gathered}
A_{n x}=q C_{n}-B_{n} r, \\
B_{n+1}=-B_{n x}+q D_{n}-A_{n} q-B_{n} \xi-D_{n} s, \\
C_{n+1}=C_{n x}-r A_{n}+D_{n} r-\xi C_{n}-s C_{n}, \\
D_{n x}-D_{n y}=r B_{n}-C_{n} q+s D_{n}-D_{n} s .
\end{gathered}
$$

Taking a few initial values as follows

$$
B_{0}=C_{0}=D_{0}=0, \quad A_{0}=-\xi^{-1}
$$

one infers from $(60)$ that

$$
\begin{gathered}
B_{1}=q \xi^{-1}-q_{y} \xi^{-2}+q_{y y} \xi^{-3}+O\left(\xi^{-4}\right), \\
C_{1}=r \xi^{-1} \\
A_{1}=\bar{A}_{1} \xi^{-2}+\widetilde{A}_{1} \xi^{-3}+O\left(\xi^{-4}\right),
\end{gathered}
$$


where $\partial_{x} \bar{A}_{1}=(q r)_{y}, \partial_{x} \widetilde{A}_{1}=-q_{y y}$, and

$$
\begin{aligned}
& D_{1 x}-D_{1 y}+\left[D_{1}, s\right]=0 \Longrightarrow D_{1}=0, \\
& B_{2}=-q+\left(-q_{x}+q_{y}-q s\right) \xi^{-1}+O\left(\xi^{-2}\right), \\
& \begin{aligned}
& C_{2}=-r+\left(r_{x}-r_{y}-s r\right) \xi^{-1}+O\left(\xi^{-2}\right), \\
& D_{2 x}-D_{2 y}+\left[D_{2}, s\right]=[\left.-(r q)_{x}+(r q)_{y}+[-r q, s]\right] \xi^{-1} \\
&+O\left(\xi^{-2}\right) \\
& \Longrightarrow D_{2}=-(r q) \xi^{-1}+O\left(\xi^{-2}\right) .
\end{aligned}
\end{aligned}
$$

Thus, in terms of step (3) of the TAH scheme and (20), we obtain the $(2+1)$-dimensional D-AKNS hierarchy

$$
u_{t_{n}}=\left(\begin{array}{c}
q \\
r \\
s
\end{array}\right)_{t_{n}}=\left(\begin{array}{c}
(\partial+s) R\left(V_{2 n}\right) \\
(\partial-s) R\left(V_{3 n}\right) \\
-2 R\left(V_{4 n, x}\right)
\end{array}\right) .
$$

When $s=0$, (64) reduces to the DS hierarchy.

When $n=2, t_{2}=t$, (64) reduces to

$$
\begin{gathered}
q_{t}=-q_{x x}+q_{x y}+(q s)_{x}-s\left(q_{x}+q_{y}+q s\right), \\
r_{t}=r_{x x}-r_{x y}-(s r)_{x}-s\left(r_{x}-r_{y}-s r\right), \\
s_{t}=2(r q)_{x} .
\end{gathered}
$$

Now we consider the Hamiltonian structure of (64). A direct computation gives

$$
\begin{gathered}
\frac{\partial U}{\partial q}=U_{q}=\left(\begin{array}{ll}
0 & 1 \\
0 & 0
\end{array}\right), \quad U_{r}=\left(\begin{array}{ll}
0 & 0 \\
1 & 0
\end{array}\right), \\
U_{s}=\left(\begin{array}{ll}
0 & 0 \\
0 & 1
\end{array}\right), \quad U_{\lambda}=\left(\begin{array}{ll}
0 & 0 \\
0 & 1
\end{array}\right), \\
\left\langle V, U_{q}\right\rangle=R\left(V_{3}\right), \quad\left\langle V, U_{r}\right\rangle=R\left(V_{2}\right), \\
\left\langle V, U_{s}\right\rangle=R\left(V_{4}\right), \quad\left\langle V, U_{\lambda}\right\rangle=4 R\left(V_{4}\right) .
\end{gathered}
$$

Substituting the above results into the trace identity (9) yields that

$$
\frac{\delta}{\delta u}\left(4 R\left(V_{4}\right)\right)=\left(\lambda^{-} \gamma \frac{\partial}{\partial \lambda} \lambda^{\gamma}\right)\left(\begin{array}{l}
R\left(V_{3}\right) \\
R\left(V_{2}\right) \\
R\left(V_{4}\right)
\end{array}\right) .
$$

Comparing the coefficients of $\lambda^{-n-1}$ reads

$$
\frac{\delta}{\delta u}\left(4 R\left(V_{4, n+1}\right)\right)=(-n+\gamma)\left(\begin{array}{l}
R\left(V_{3 n}\right) \\
R\left(V_{2 n}\right) \\
R\left(V_{4 n}\right)
\end{array}\right) .
$$

Inserting the initial values in (60) gives $\gamma=0$. Thus, we have

$$
\left(\begin{array}{l}
R\left(V_{3 n}\right) \\
R\left(V_{2 n}\right) \\
R\left(V_{4 n}\right)
\end{array}\right)=\frac{\delta}{\delta u}\left(-\frac{4 R\left(V_{4, n+1}\right)}{n}\right) \equiv \frac{\delta H_{n}}{\delta u} .
$$

Therefore, the $(2+1)$-dimensional D-AKNS hierarchy (64) can be written as the Hamiltonian form

$$
u_{t_{n}}=\left(\begin{array}{c}
q \\
r \\
s
\end{array}\right)_{t_{n}}=J \frac{\delta H_{n}}{\delta u}
$$

where

$$
J=\left(\begin{array}{ccc}
0 & \partial+s & 0 \\
\partial-s & 0 & 0 \\
0 & 0 & -2 \partial
\end{array}\right)
$$

In what follows, we want to discuss the $(2+1)$-dimensional expanding models of the $(2+1)$-dimensional D-AKNS hierarchy (64). Employing the loop algebra of the Lie algebra $G_{2}$ introduces the following Lax pair:

$$
\begin{gathered}
U=\left(\begin{array}{cccc}
0 & q & 0 & v_{1} \\
r & \lambda+\xi+s & v_{2} & 0 \\
0 & v_{1} & 0 & q \\
v_{2} & 0 & r & \lambda+\xi+s
\end{array}\right), \\
V=\left(\begin{array}{ll}
A & B \\
B & A
\end{array}\right)
\end{gathered}
$$

where

$$
\begin{gathered}
A=\left(\begin{array}{ll}
V_{1} & V_{2} \\
V_{3} & V_{4}
\end{array}\right), \quad B=\left(\begin{array}{ll}
V_{5} & V_{7} \\
V_{8} & V_{6}
\end{array}\right), \\
V_{i}=\sum_{m \geq 0} V_{i m} \lambda^{-m}, \quad i=1,2, \ldots, 8 .
\end{gathered}
$$

Solving the matrix-operator equation (6) yields that

$$
\begin{aligned}
& V_{1 x}=q V_{3}+v_{3} V_{5}+v_{1} V_{8}-V_{2} r-V_{5} v_{3}-V_{7} v_{2}, \\
& V_{2 x}=q V_{4}+v_{3} V_{7}+v_{1} V_{6}-V_{1} q-\lambda V_{2}-V_{2} \xi-V_{2} s-V_{5} v_{1}, \\
& V_{5 x}=q V_{8}+v_{3} V_{1}+v_{1} V_{3}-V_{1} v_{3}-V_{2} v_{2}-V_{7} r, \\
& V_{7 x}=q V_{6}+v_{3} V_{2}+v_{1} V_{4}-V_{1} v_{1}-V_{5} q-V_{7} \lambda-V_{7} \xi-V_{7} s \\
& V_{3 x}=r V_{1}+\lambda V_{3}+\xi V_{3}+s V_{3}+v_{2} V_{5}-V_{4} r-V_{8} v_{3}-V_{6} v_{2}, \\
& V_{4 x}=r V_{2}+\xi V_{4}+s V_{4}+v_{1} V_{7}-V_{3} q-V_{4} \xi-V_{4} s-V_{8} v_{1}, \\
& V_{8 x}=r V_{5}+\lambda V_{8}+\xi V_{8}+s V_{8}+v_{2} V_{1}-V_{3} v_{3}-V_{4} v_{2}-V_{6} r \\
& V_{6 x}=r V_{7}+\xi V_{6}+s V_{6}+v_{2} V_{2}-V_{3} v_{1}-V_{8} q-V_{6} \xi-V_{6} s .
\end{aligned}
$$

Substituting (73) into (74) gets that

$$
\begin{aligned}
& V_{1 n, x}=q V_{3 n}+v_{1} V_{8 n}-V_{2 n} r-V_{7 n} v_{2}, \\
& V_{2, n+1}=-V_{2 n, x}+q V_{4 n}+v_{1} V_{6 n}-V_{1 n} q-V_{2 n} \xi-V_{2 n} s-V_{5 n} v_{1},
\end{aligned}
$$




$$
\begin{aligned}
& V_{5 n, x}=q V_{8 n}+v_{1} V_{3 n}-V_{2 n} v_{2}-V_{7 n} r, \\
& V_{7, n+1}=-V_{7 n, x}+q V_{6 n}+v_{1} V_{4 n}-V_{1 n} v_{1}-V_{5 n} q-V_{7 n} \xi-V_{7 n} s, \\
& V_{3, n+1}=\partial_{-} V_{3 n}-r V_{1 n}-V_{3 n} \xi-s V_{3 n}-v_{2} V_{5 n}+V_{4 n} r+V_{6 n} v_{2}, \\
& V_{4 n, x}=r V_{2 n}+V_{4 n, y}+s V_{4 n}+v_{2} V_{7 n}-V_{3 n} q-V_{4 n} s-V_{8 n} v_{1}, \\
& V_{8, n+1}=\partial_{-} V_{8 n}-r V_{5 n}-V_{8 n} \xi-s V_{8 n}-v_{2} V_{1 n}+V_{4 n} v_{2}+V_{6 n} r, \\
& V_{6 n, x}=r V_{7 n}+V_{6 n, y}+s V_{6 n}+v_{2} V_{2 n}-V_{3 n} v_{1}-V_{8 n} q-V_{6 n} s,
\end{aligned}
$$

where $\partial_{-}=\partial_{x}-\partial_{y}$.

Taking

$$
\begin{gathered}
V_{2,0}=V_{3,0}=V_{4,0}=V_{6,0}=V_{7,0}=V_{8,0}=0, \\
V_{1,0}=V_{5,0}=-\xi^{-1},
\end{gathered}
$$

then one infers from (60) that

$$
\begin{aligned}
V_{2,1}= & \left(q+v_{1}\right) \xi^{-1}-\left(v_{1 y}+q_{y}\right) \xi^{-2}+\left(q_{y y}+v_{1 y y}\right) \xi^{-3} \\
& +O\left(\xi^{-4}\right), \\
V_{3,1}= & V_{8,1}=\left(r+v_{2}\right) \xi^{-1}, \\
V_{7,1}= & \left(q+v_{1}\right) \xi^{-1}-\left(v_{1 y}+q_{y}\right) \xi^{-2}+\left(v_{1 y y}+q_{y y}\right) \xi^{-3} \\
& +O\left(\xi^{-4}\right), \\
V_{1,1}= & \bar{V}_{1,1} \xi^{-2}+O\left(\xi^{-3}\right),
\end{aligned}
$$

where $\partial_{x} \bar{V}_{1,1}=\left(q r+v_{1} r+v_{1} v_{2}+q v_{2}\right)_{y}$,

$$
V_{5,1}=\bar{V}_{5,1} \xi^{-2}+O\left(\xi^{-3}\right)
$$

where $\partial_{x} \bar{V}_{5,1}=\left(v_{1} v_{2}+v_{1} r+q r+q v_{2}\right)_{y}$,

$$
\begin{gathered}
\left(V_{4,1}\right)_{x}+\left[V_{4,1}, s\right]=0 \Longrightarrow V_{4,1}=0, \\
\left(V_{6,1}\right)_{x}+\left[V_{6,1}, s\right]=0 \Longrightarrow V_{6,1}=0, \\
V_{2,2}=-q-v_{1}-\left(q_{x}-q_{y}+v_{1 x}-v_{1 y}-q s-v_{1} s\right) \xi^{-1} \\
+O\left(\xi^{-2}\right), \\
V_{7,2}=\left(-v_{1 x}-q_{x}+v_{1 y}+q_{y}-v_{1} s-q s\right) \xi^{-1}+O\left(\xi^{-2}\right), \\
V_{3,2}=V_{8,2}=-r-v_{2}+\left(r_{x}+v_{2 x}-r_{y}-v_{2 y}-s r-s v_{2}\right) \xi^{-1} \\
+O\left(\xi^{-2}\right),
\end{gathered}
$$

$$
\begin{aligned}
\left(V_{4,2}\right)_{x}-\left(V_{4,2}\right)_{y}+\left[V_{4,2}, s\right] & \\
= & -\left(r q+r v_{1}+v_{2} v_{1}+v_{2} q\right)_{x} \xi^{-1} \\
& +\left(r q+r v_{1}+v_{2} v_{1}+v_{2} q\right)_{y} \xi^{-1} \\
& +\left[\left(r q+r v_{1}+v_{1} v_{1}+v_{2} q\right) s\right. \\
& \left.\quad-s\left(r q+v_{2} q+r v_{1}+v_{2} v_{1}\right)\right] \xi^{-1}+O\left(\xi^{-2}\right) \\
\Longrightarrow & V_{4,2}=-\left(r q+r v_{1}+v_{2} v_{1}+v_{2} q\right) \xi^{-1} .
\end{aligned}
$$

According to step (3) of the TAH scheme and (56), we obtain the $(2+1)$-dimensional equation hierarchy

$$
u_{t_{n}}=\left(\begin{array}{c}
q \\
r \\
s \\
v_{1} \\
v_{2}
\end{array}\right)_{t_{n}}=J_{3}\left(\begin{array}{c}
2 R\left(V_{3 n}\right) \\
2 R\left(V_{2 n}\right) \\
2 R\left(V_{4 n}\right) \\
2 R\left(V_{8 n}\right) \\
2 R\left(V_{7 n}\right)
\end{array}\right),
$$

where $J_{3}$ appears in (56).

When $n=2, t_{2}=t,(80)$ reduces to the following equations:

$$
\begin{gathered}
q_{t}=-q_{x x}+q_{x y}-v_{1 x x}+v_{1 x y}+\left(q s+v_{1} s\right)_{x}-s q_{x}-s q_{y} \\
-s v_{1 x}+s v_{1 y}+s q s+s v_{1} s, \\
r_{t}=r_{x x}-r_{x y}+v_{2 x x}-v_{2 x y}-\left(s r+s v_{2}\right)_{x}-s r_{x}-s v_{2 x}+s r_{y} \\
+s v_{2 y}+s^{2} r+s^{2} v_{2}, \\
s_{t}=2\left(r q+r v_{1}+v_{2} v_{1}+v_{2} q\right)_{x}, \\
\left(v_{1}\right)_{t}=-q_{x x}+q_{x y}-v_{1 x x}+v_{1 x y}-\left(v_{1} s+q s\right)_{x}-s v_{1 x}-s q_{x} \\
+s v_{1 y}+s q_{y}-s v_{1} s-s q s, \\
\left(v_{2}\right)_{t}=r_{x x}-r_{x y}+v_{2 x x}-v_{2 x y}-\left(s r+s v_{2}\right)_{x}-s r_{x}-s v_{2 x} \\
+s r_{y}+s v_{2 y}+s^{2} r+s^{2} v_{2} .
\end{gathered}
$$

We can regard (81) as a kind of expanding model of (65). That is, when letting $s=v_{1}=v_{2}=0$, the previous three equations of (81) are just right (65). Therefore, when $s=v_{1}=$ $v_{2}=0,(80)$ can be regarded as a united model of the $(2+1)$ dimensional D-AKNS hierarchy and the DS hierarchy.

Remark. Seeking the Hamiltonian structure of (80) is our ineresting problem. However, if we apply the trace identity (9) to deduce its Hamiltonian structure, we find that it is the same with that in (70). We guess this is not a right result. In forthcoming days, we will go on to consider the problem.

\section{Conflict of Interests}

The authors declare that there is no conflict of interests regarding the publication of this paper. 


\section{Acknowledgment}

This work was supported by the Natural Science Foundation of Shandong Province (Grant no. ZR2013AL016) and the National Natural Science Foundation of China (Grant no. 11371361) as well as the Fundamental Research Funds for the Central Universities (2013XK03).

\section{References}

[1] F. Magri, "A simple model of the integrable Hamiltonian equation," Journal of Mathematical Physics, vol. 19, no. 5, pp. 1156-1162, 1978.

[2] G. Z. Tu, "The trace identity, a powerful tool for constructing the Hamiltonian structure of integrable systems," Journal of Mathematical Physics, vol. 30, no. 2, pp. 330-338, 1989.

[3] W. X. Ma, "A hierarchy of Liouville integrable generalized Hamiltonian equations and its reduction," Chinese Journal of Contemporary Mathematics, vol. 13, no. 1, pp. 79-89, 1992.

[4] W. X. Ma, "A hierarchy of Liouville integrable finite-dimensional Hamiltonian systems," Applied Mathematics and Mechanics, vol. 13, no. 4, pp. 369-377, 1992.

[5] W. Ma, "An approach for constructing non-isospectral hierarchies of evolution equations," Journal of Physics A: Mathematical and General, vol. 25, no. 12, pp. L719-L726, 1992.

[6] W. Ma, "A Hamiltonian structure associated with a matrix spectral problem of arbitrary-order," Physics Letters. A, vol. 367, no. 6, pp. 473-477, 2007.

[7] X. B. Hu, "A powerful approach to generate new integrable systems," Journal of Physics A: Mathematical and General, vol. 27, no. 7, pp. 2497-2514, 1994.

[8] X. Hu, "An approach to generate superextensions of integrable systems," Journal of Physics A: Mathematical and General, vol. 30, no. 2, pp. 619-632, 1997.

[9] E. Fan, "A family of completely integrable multi-Hamiltonian systems explicitly related to some celebrated equations," Journal of Mathematical Physics, vol. 42, no. 9, pp. 4327-4344, 2001.

[10] E. G. Fan, "Integrable evolution systems based on GerdjikovIvanov equations, bi-Hamiltonian structure, finite-dimensional integrable systems and $N$-fold Darboux transformation," Journal of Mathematical Physics, vol. 41, no. 11, pp. 7769-7782, 2000.

[11] S. Chakravarty, S. L. Kent, and E. T. Newman, "Some reductions of the self-dual Yang-Mills equations to integrable systems in $2+1$ dimensions," Journal of Mathematical Physics, vol. 36, no. 2, pp. 763-772, 1995.

[12] M. J. Ablowitz, S. Chakravarty, and L. A. Takhtajan, "A self-dual Yang-Mills hierarchy and its reductions to integrable systems in $1+1$ and $2+1$ dimensions," Communications in Mathematical Physics, vol. 158, no. 2, pp. 289-314, 1993.

[13] G. Z. Tu, R. I. Andrushkiw, and X. C. Huang, "A trace identity and its application to integrable systems of $1+2$ dimensions," Journal of Mathematical Physics, vol. 32, no. 7, pp. 1900-1907, 1991.

[14] G. Z. Tu and D. Z. Meng, “The trace identity, a powerful tool for constructing the Hamiltonian structure of integrable systems (II)," Acta Mathematicae Applicatae Sinica, vol. 5, no. 1, pp. 8996, 1989.

[15] W. Ma and M. Chen, "Hamiltonian and quasi-Hamiltonian structures associated with semi-direct sums of Lie algebras," Journal of Physics A: Mathematical and General, vol. 39, no. 34, pp. 10787-10801, 2006. 


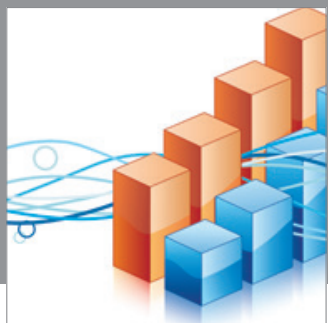

Advances in

Operations Research

mansans

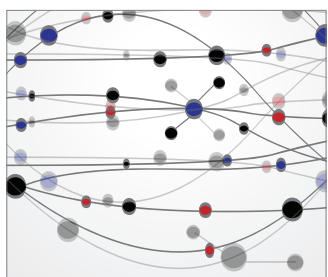

The Scientific World Journal
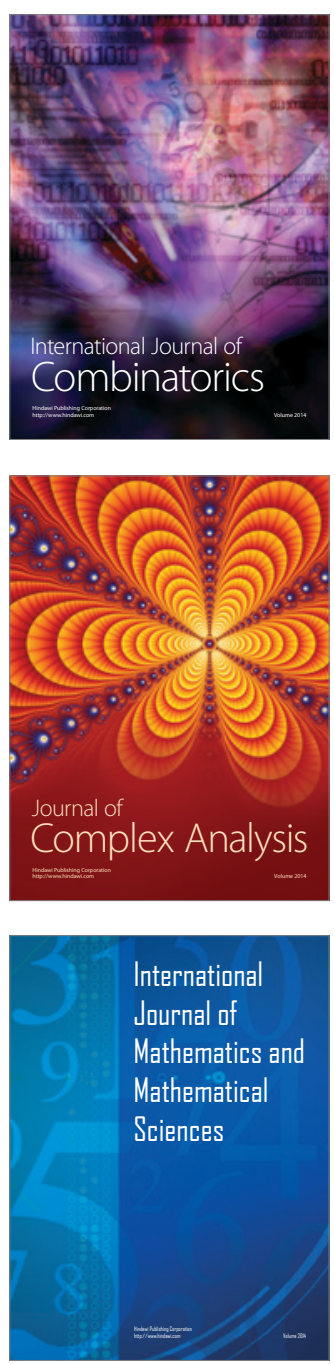
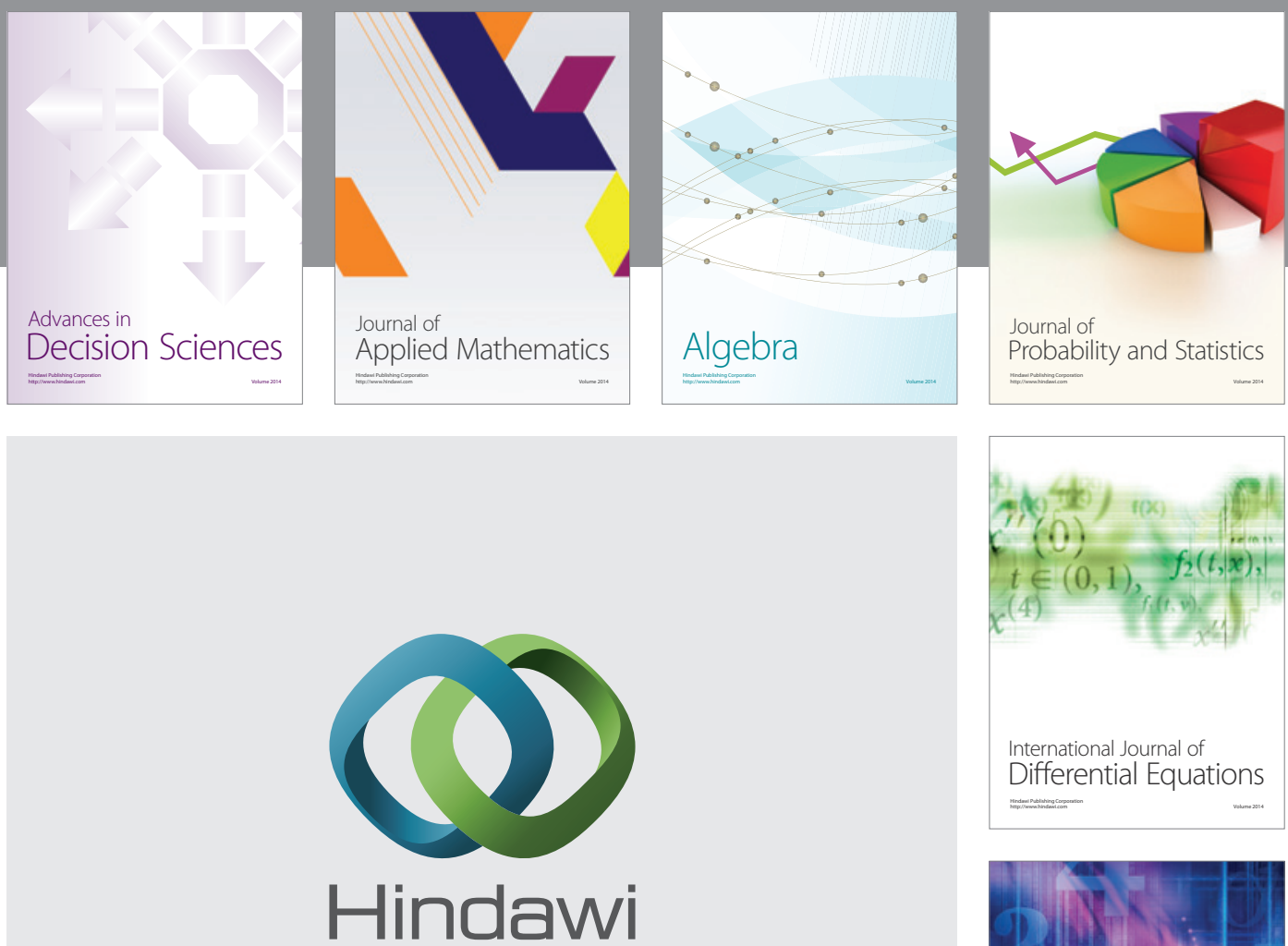

Submit your manuscripts at http://www.hindawi.com
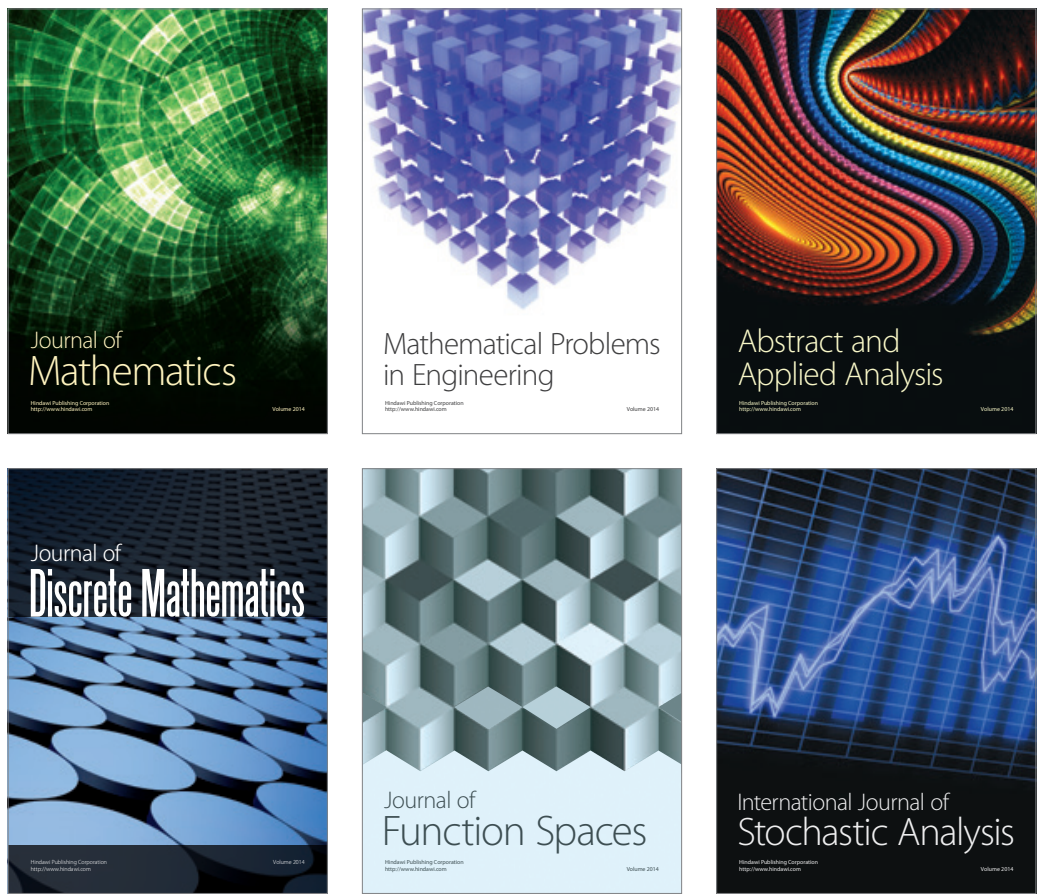

Journal of

Function Spaces

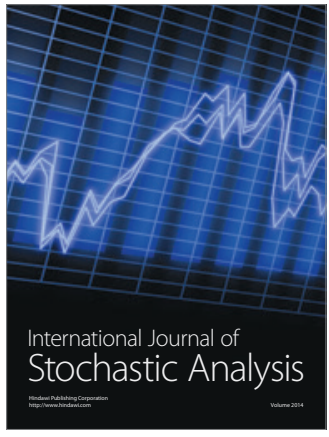

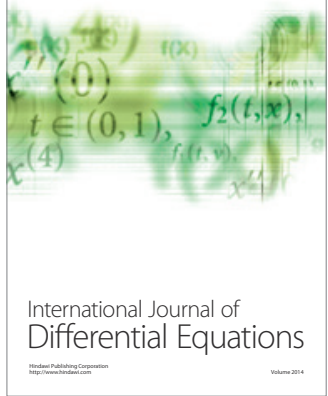
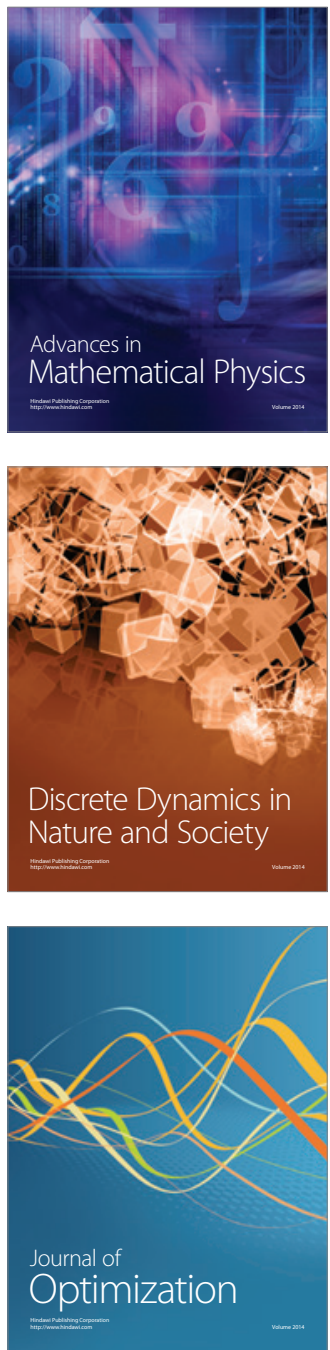